\title{
ADAPTIVE QUANTISATION IN HEVC FOR CONTOURING ARTEFACTS REMOVAL IN UHD CONTENT
}

\author{
Nicolò Casali ${ }^{1,2}$,Matteo Naccari $^{1}$, Marta Mrak $^{1}$ and Riccardo Leonardi ${ }^{2}$ \\ ${ }^{1}$ British Broadcasting Corporation - Research and Development, London, United Kingdom \\ ${ }^{2}$ Università degli Studi di Brescia, Brescia, Italy
}

\begin{abstract}
Contouring artefacts affect the visual experience of some particular types of compressed Ultra High Definition (UHD) sequences characterised by smoothly textured areas and gradual transitions in the value of the pixels. This paper proposes a technique to adjust the quantisation process at the encoder so that contouring artefacts are avoided. The devised method does not require any change at the decoder side and introduces a negligible coding rate increment (up to $3.4 \%$ for the same objective quality). This result compares favourably with the average $11.2 \%$ bit-rate penalty introduced by a method where the quantisation step is reduced in contour-prone areas.
\end{abstract}

Index Terms - Contouring artefact, video coding, HEVC, adaptive dead zone adjustment

\section{INTRODUCTION}

With the advent of higher resolution cameras and displays, users will be provided with a more immersive and compelling experience when watching television programmes. The format which will deliver this improved quality of experience is called Ultra High Definition Television (UHDTV). The volume of data associated with this new format is eight times the one associated with High Definition Television (HDTV) and therefore efficient compression technology should be employed to guarantee that UHDTV signals can be delivered using current networks. Lossy video compression (i.e. the one commonly used in broadcasting and streaming applications) inevitably lowers the quality of the uncompressed content by introducing coding artefacts. Block-based video codecs like the ones specified by High Efficiency Video Coding (HEVC, [1]) introduce different types of artefacts; the most prominent are blocking, ringing and blurring [2]. Moreover, in image areas with low contrast and smooth variation of pixel intensities, the contouring or banding artefact may also appear with false edges located along pixel variations.

Historically contouring was mainly associated with the reduced dynamic range of displays [3, 4] which, for implementation costs, offered less than 8 bits per component. Nowadays with the advent of high dynamic range displays at affordable prices for consumers, contouring is no longer caused by bit depth limitation for standard dynamic range material (i.e. 8 bits per color component). However, contouring may still appear in low contrast image areas after compression. In fact, in these regions both intra and inter coding modes can efficiently predict the original pixels values and provide a residual signal with low energy and thus few frequency coefficients with small magnitude which will be then rounded to zero leading to the creation of banding effects.

The HEVC syntax allows the quantisation step to be varied for a given coding unit so that contour-prone areas may be quantized with low step values and false edges are prevented. However, from what stated above, the quantisation step should be quite small to avoid small magnitude coefficients being rounded to zero. This will result in an increased bit-rate which is not desirable in many application scenarios. It should be also noted that given the increased resolution associated with UHD material, contouring artefacts will be more noticeable over UHDTV displays. Thus, the perceptual quality of image areas affected by contouring should be optimised to meet the high expectations of UHDTV users.

In this context, this paper proposes an adaptive quantisation method to prevent contouring artefacts in UHD video coded with the HEVC standard. The main idea is to vary the quantiser deadzone in areas affected by contouring so that some coefficients are not rounded to zero and false edges are avoided. The paper also presents an analysis of the coefficient distribution over image blocks associated with contouring and uses this analysis to decide over which coefficients the dead-zone should be modified.

The remainder of this paper is organised as follows. Section 2 provides an overview on the relevant literature for contouring artefacts. An analysis of the coefficient distribution over blocks potentially affected by contouring as well as the proposed adaptive quantisation is presented in Section 3. Section 4 provides the performance analysis for the proposed method while conclusions and future work are pointed out in Section 5.

\section{RELATED WORK}

All state-of-the-art methods differentiate by whether contouring removal (hereafter also denoted as decontouring) is applied at the encoder side (pre-processing) or at the decoder side (post-processing). This section provides an overview on the methods proposed in the literature for decontouring starting with pre-processing.

One of the pioneering pre-processing methods for decontouring is the one proposed by Roberts [5]. The method is applied to pulse code modulation for image coding and adds a pseudo-random noise to the image prior to quantisation to overcome bit-depth limitations of displays. At the decoder, the noise is subtracted to the image before displaying. The method efficiently removed contouring artefacts when compressing 6 bit per pixel black and white images to 2-3 bits per pixels. Daly et al. in [4] extended Roberts' work by proposing Bit-Depth Extension (BDE), a dithering process where the noise addition is shaped using a spatio-temporal model of human contrast sensitivity function. This BDE method can efficiently hide contouring artefacts without compromising the whole picture quality. During the development of the HEVC standard, contouring was also addressed. The work in [6] identifies edges across pixels used to compute intra predictors as the main cause for contouring. In fact, these edges will then propagate in the residual leading to 
banding. To avoid this, the solution proposed is to apply bilinear interpolation to the samples used for prediction and only over $32 \times 32$ coding blocks where the artefact is more noticeable.

There have been many proposals for post-processing decontouring, i.e. applied after decoding. The work from Yoo et al. in [7] proposed to perform dithering by adding random noise inside the coding loop after each contour-prone block is reconstructed. The noise is added on a macroblock basis using patterns which are then transmitted to the receiver to avoid mismatches between encoder and decoder. This in-loop dithering is implemented in the H.264/AVC reference implementation (JM) and subjective tests were run to compare the perceived quality improvement. For an average $0.33 \%$ of coding penalty, the method increases the average mean opinion score by 0.71 . Still using dithering, the work by Bhagavathy et al. [8] analyses the decoded image at different resolutions (scales) and adds random noise to hide false contours. Visual inspection over images processed by this technique reveals that the visibility of false edges is efficiently reduced. Jin et al. [9] and Wang et al. [10] proposed similar block-based dithering techniques, adjusting the average value of each block to improve the gradient smoothness. Contouring can also be removed by filtering along the direction of false edges as proposed by Lee et al. in [11]. The devised filtering provides better subjective quality with respect to the other methods considered for comparison (including [4]). Following the idea of directional filtering, Choi et al. in [12] proposed to apply weighted smoothing filters to false edges. The method provides better subjective quality and higher PSNR values with respect to its competitors. The method proposed by Ahn et al. in [13] randomly shuffles the pixel position over blocks affected by contouring and then applies low pass filtering to remove false edges. Still applying filtering, the work from Daly and Feng [14] detects false contours by applying low pass filtering to an extended bit-depth version of the decoded image. The highlighted false edges are then subtracted from the decoded image to remove contouring.

\section{PROPOSED ADAPTIVE QUANTISATION}

This section presents the proposed adaptive quantisation for decontouring in UHD video. The analysis of the frequency coefficient distribution is detailed first followed by the design of the adaptive dead-zone adjustment. Finally, the overall algorithm is presented.

\subsection{Analysis of coefficient distribution for contour-prone blocks}

As stated in the Introduction, contouring mainly affects image areas with low contrast and smooth gradient variations. As an example, Fig. 1(a) shows the first frame of the Ningyo sequence belonging to the test set used in this work. Fig. 1(b) shows the pixel variations for the luma component in the highlighted red box where the color map has been changed to highlight different pixel values. For the $\mathrm{YC}_{b} \mathrm{C}_{r}$ color space, only the luma $Y$ component is affected by contouring, therefore the following analysis and the proposed adaptive quantisation will hereafter refer to this component. Fig. 1(c) shows the same area of Fig. 1(b) coded with the HEVC reference Model (HM) and quantisation Parameter (QP) equal to 27. As may be noted, false edges associated with contouring artefacts are now clearly visible. Given the smooth pixel transitions in contour-prone blocks, the encoder tends to apply large transforms (e.g. $32 \times 32$ or $16 \times 16)$ to compress these areas since they will result in few frequency coefficients, rounded to zero after quantisation.

The proposed adaptive quantisation varies the quantiser deadzone so that some coefficients are not rounded to zero and smooth gradient transitions can be preserved. One key aspect to be addressed

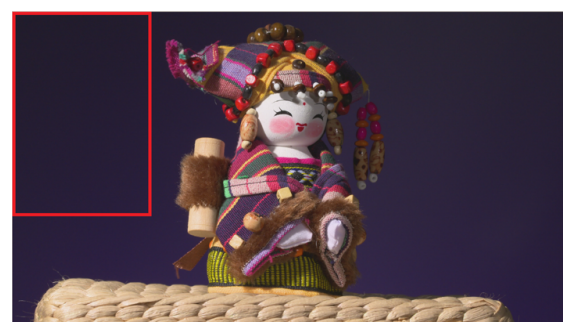

(a) Original frame

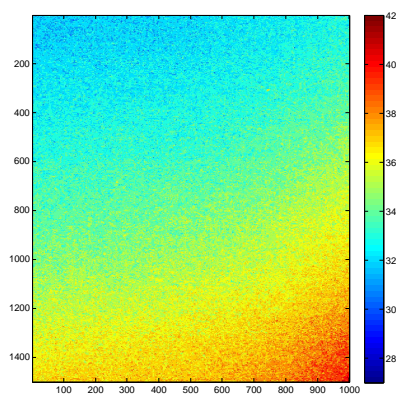

(b) Original frame

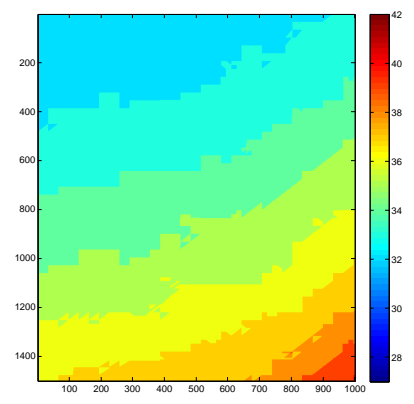

(c) Coded frame
Fig. 1. Example of contouring artefact for the Ningyo sequence.

in the design is which coefficients should be selected for this adjustment. Accordingly, extensive simulations were run over the Ningyo and YoungDancers sequences since they contain large areas affected by contouring, allowing meaningful results and statistics to be collected. The HM codec with QP values in $\{22,27,32,37\}$ and the intra main configuration [15] were used. To detect contour-prone blocks, the detector described in [10] is used. For block sizes of $8 \times 8,16 \times 16$ and $32 \times 32$ the dead-zone of the quantiser is varied over contour-prone blocks and for coefficients whose radial frequency $f_{r}$ satisfies the following condition:

$$
f_{r}(i, j)=\sqrt{i^{2}+j^{2}} \leq B / 4,
$$

where $B$ denotes the block size and $i, j$ are the DCT bands. The quantiser dead-zone is varied so it results between $13 \%$ and $75 \%$ of the size used by HM. After compression, each coded sequence has been visually inspected to assess whether contouring was still noticeable. The coefficients which prevent contouring and minimise the additional bit-rate are shown in the shaded areas of Fig. 2. The selection of these coefficients can be also motivated by analysing the spectral density of residuals associated with contour-prone blocks. More precisely, a simple first order autoregressive model is used to predict the value of each residual $r$ at location $(x, y), y$ representing the row direction:

$$
r(x, y)=\alpha \cdot r(x, y-1)+z\left(\mu, \sigma_{z}^{2}\right),
$$

where $\alpha$ is the correlation coefficient in the range $[0,1]$ and $z$ is assumed to be a white Gaussian noise with mean $\mu$ and variance $\sigma_{z}^{2}$. Parameters $\alpha, \mu$ and $\sigma_{z}^{2}$, are estimated from the residuals collected for different block sizes and QP values over the sequences tested. The power spectral density $S(f)$ is then computed as:

$$
S(f)=\frac{\sigma_{z}^{2}}{\left|1-\alpha \cdot e^{-2 j \pi f}\right|^{2}},
$$

where the shape of $S(f)$ is determined by $\alpha$, that is, the closer to 1 the more energy is concentrated in the low frequencies. For the 

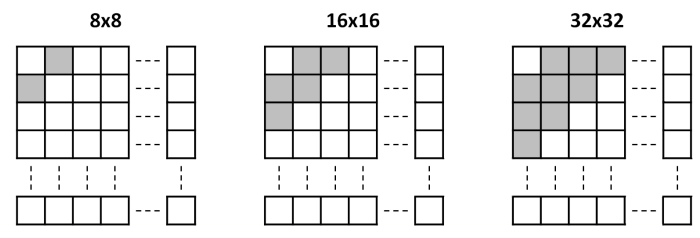

Fig. 2. Selected coefficients for dead-zone adjustment.

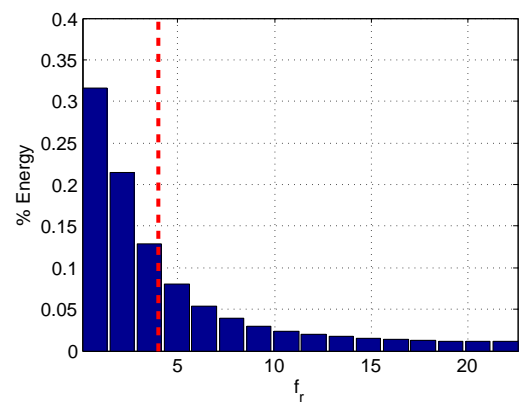

Fig. 3. Percentage of energy for each radial frequency.

collected data, the value for $\alpha$ was found to be in the range of [0.6, 0.7 ] for all considered block sizes. With this value, up to $65 \%$ of the whole energy is concentrated in the frequency range $[0, B / 4]$. Fig. 3 shows the percentage of energy contained for each radial frequency value in a $16 \times 16$ block. The red dashed line refers to the $65 \%$ limit attained by summing the energy for bands whose frequency is lower than $B / 4$. This analysis shows how the selected coefficients in Fig. 2 characterize smooth gradient variations in image areas affected by contouring.

\subsection{Adaptive variation of quantiser dead-zone}

HEVC adopts a dead-zone scalar uniform quantiser whereby for each coefficient $c$ at frequency $(i, j)$, the corresponding quantisation level $l(i, j)$ is given by:

$$
l(i, j)=\operatorname{sign}\{c(i, j)\} \cdot\left\lfloor\frac{|c(i, j)|+\theta}{\Delta}\right\rfloor,
$$

where $\operatorname{sign}\{\cdot\}$ denotes the sign function, $\Delta$ the quantisation step, $\theta$ the rounding offset and $\lfloor\cdot\rfloor$ returns the nearest integer less or equal to its argument. Offset $\theta$ controls the extent of the dead-zone, which is the decision interval with reconstruction value equal to zero (see Fig. 4). As stated in Section 3.1, most of the coefficients, namely those shaded in Fig. 2, are rounded to zero given their small magnitude. Therefore, the proposed method adjusts the size of the dead-zone for these coefficients so that they will be quantized to one of the two levels adjacent to the dead-zone. The adjustment is not performed for $4 \times 4$ blocks as contour-prone areas are coded with larger block sizes. For all the remaining coefficients the value for $\theta$ is set to the default one used by HM, that is $1 / 3$ or $1 / 6$ for intra and inter slices, respectively. In the proposed adaptive quantisation, the value for $\theta$ is selected on a Transform Block (TB, [1]) basis from a set of predefined values in the range $\Theta=[0.5,0.8]$ spaced by a step $s=$ 0.05 . The optimal offset is selected by counting the number of non zero levels as described in the following sequence of steps:

1. Set $\theta$ to $\min (\Theta)$

2. Apply (4) to all selected coefficients as in Fig. 2
3. Count the number, $n$, of non zero levels obtained

4. If $n \geq \tau$ then terminate else set $\theta=\theta+s$ and go to Step 2

In this paper, the value for $\tau$ is set to one. Moreover, over TBs affected by contouring, the Rate Distortion optimised quantisation (RDOQ, [16]) is disabled since the cost function used does not take into account contouring artefacts.

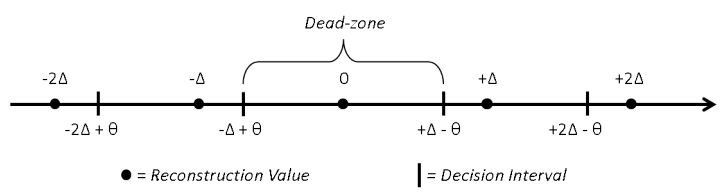

Fig. 4. Uniform scalar dead-zone quantiser.

\subsection{Overall system}

The block scheme for the proposed adaptive quantisation is shown in Fig. 5. Accordingly, the luma component of each coded frame is used to detect contour-prone image areas. In this paper, the detector proposed in [10] is used and the detection is carried out using a $64 \times 64$ block granularity. The detector generates a binary map where each entry indicates whether the corresponding $64 \times 64$ block will be affected by contouring. This map is then used in the quantisation stage to decide the value for offset $\theta$ as described in Section 3.2. From the classification given in Section 2, it should be noted that the proposed method is placed amid pre-processing and post-processing methods as it operates inside the coding process. However, the proposed adaptive quantisation is completely transparent to the decoder as the quantized levels are still compliant with the HEVC syntax.



Fig. 5. Block scheme of the proposed adaptive quantisation.

\section{EXPERIMENTAL RESULTS}

This section presents the assessment made to validate the proposed adaptive quantisation for decontouring. The test material and coding conditions are presented first, followed by the performance indicators. The results obtained will then be reported and commented.

\subsection{Test material and coding conditions}

The test set is composed of five sequences with 8 bits per component, 4:2:0 chroma format, $3840 \times 2160$ spatial resolution and frame rate of 50 and $60 \mathrm{fps}$. These sequences are denoted as "Badminton", "CandleSmoke", "Ningyo", "ShowDrummer" and "YoungDancers". The thumbnail of the first frame for each sequence is shown in Fig. 6. The test is composed by content representative of broadcasting 


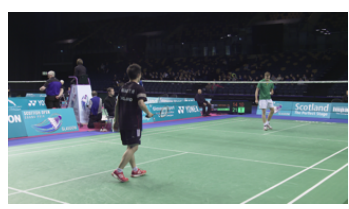

(a)

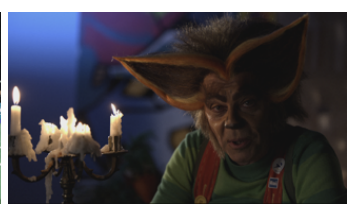

(b)

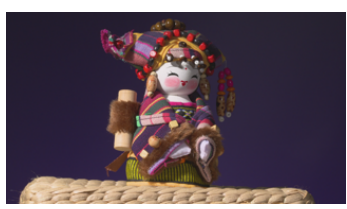

(c)



(d)

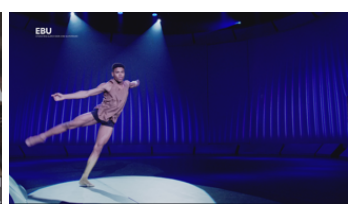

(e)

Fig. 6. Sequences from the test set: (a) Badminton, (b) CandleSmoke, (c) Ningyo, (d) ShowDrummer and (e) YoungDancers.

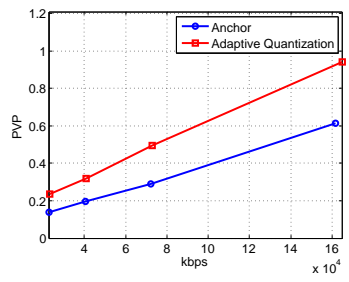

(a)

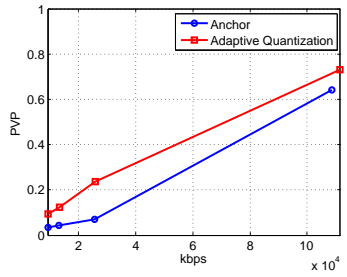

(b)

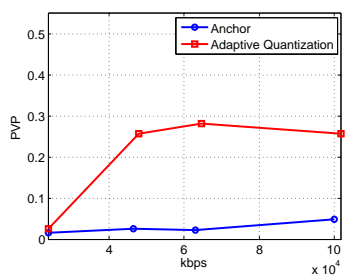

(c)



(d)

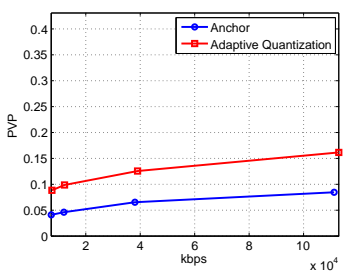

(e)

Fig. 7. Rate and PVP score charts: (a) Badminton, (b) CandleSmoke, (c) Ningyo, (d) ShowDrummer and (e) YoungDancers.

(e.g. Badminton and ShowDrummer) with all sequences containing large areas where contouring artefacts may be introduced. These five sequences have been encoded with the HM encoder (Version 12.0) and according to the intra main configuration. QP values are $\{22$, $27,32,37\}$ as suggested in [15].

\subsection{Performance indicators and benchmarks}

The performance of the proposed method is assessed in terms of coding efficiency penalty and image quality improvement. The coding efficiency penalty is measured by using the Bjøntegaard Delta-rate (BD-rate, [17]) computed over the luma component only between each video coded with HM and with HM plus the proposed method. For picture quality, the preventions of contouring artefacts needs to be quantified by checking how much small pixel variations are preserved in coded images. This can be achieved by computing, for each contour-prone block $c$, the discrete derivative $d_{\phi}(c)$ using one pixel offset and along direction $\phi$, with $\phi$ being either horizontal (hor) or vertical (ver). For frame $F$, the Pixel Variation Preservation (PVP) score is defined as:

$$
P V P(F)=\frac{1}{2 N(F)} \sum_{i=0}^{N(F)}\left(\frac{d_{\text {hor }}(\hat{c}(i))}{d_{\text {hor }}(c(i))}+\frac{d_{\text {ver }}(\hat{c}(i))}{d_{\text {ver }}(c(i))}\right),
$$

where $c(i)$ and $\hat{c}(i)$ denote the $i$-th contour-prone block in the original and coded frames, respectively and $N(F)$ is the number of all contour-prone blocks in $F$. At sequence level, the PVP score is given as the average across all coded frames. It should be noted that when contouring is absent the value for PVP is close to one and is close to zero when contouring is present. Finally, to further compare the performance of the proposed method, the HM codec has been also modified so that, for each contour-prone block, RDOQ is disabled and the QP is lowered by a given amount selected for each video so that contouring was prevented in the reconstructed pictures. This method will be hereafter denoted as Decontouring- $Q P$.

\subsection{Results and analysis}

Table 1 reports the BD-rate values provided by the proposed method and the Decontouring- $Q P$. All coded sequences have been also vi-
Table 1. BD-rate for the proposed method and Decontouring- $Q P$.

\begin{tabular}{|c|c|c|}
\cline { 2 - 3 } \multicolumn{1}{c|}{} & \multicolumn{2}{c|}{ Y BD-rate [\%] } \\
\hline Sequence & Proposed & Decontouring-QP \\
\hline Badminton & 0.5 & 3.9 \\
CandleSmoke & 0.5 & 16.6 \\
Ningyo & 3.4 & 11.7 \\
ShowDrummer & 2.7 & 13.9 \\
YoungDancers & 2.3 & 10.0 \\
\hline Average & 1.9 & 11.2 \\
\hline
\end{tabular}

sually inspected to ensure that no noticeable contouring was present in the content coded with both methods. From the reported values it may be seen that the proposed method can prevent contouring by requiring a small BD-rate penalty of up $3.4 \%$. These results compare favourably with the Decontouring- $Q P$ method which requires up to $16.6 \%$ rate increase to achieve the same perceptual quality. Fig. 7 reports the rate vs PVP score charts obtained over the test content for both the anchor and the proposed method. As a first observation, the PVP metric satisfies the expectation where the higher the bit-rate, the more pixel variation is preserved. For ShowDrummer scores higher than one are obtained since quantisation added more variation to the coded sequence without influencing the overall quality. Moreover, for Ningyo a slightly score drop corresponding to QP 22 versus QP 27 is observed, although below the noise level. Overall, it can be concluded that what observed by visual inspection is also confirmed by the curves in Fig. 7 .

\section{CONCLUSIONS AND FUTURE WORK}

This paper proposed an adaptive quantisation which adjusts the quantiser dead-zone to prevent contouring artefacts. The method can avoid false edges in video coded with HEVC at negligible BDrate penalties. Future work will develop along two main directions: extension to inter coding, addressing drift propagation due to motion compensation. Second a formal subjective evaluation campaign will be performed to confirm the perceived quality provided by the method and that PVP metric evolves consistently with MOS values. 


\section{REFERENCES}

[1] ISO/IEC and ITU-T, Information technology - High efficiency coding and media delivery in heterogeneous environments - Part 2: High efficiency video coding and H.265: High Efficiency Video Coding, Nov. 2013.

[2] M. Yuen and H. R. Wu, "A survey of hybrid MC/DPCM/DCT video coding distortions," Signal Processing, vol. 70, no. 3, pp. 247-278, Nov. 1998.

[3] T. Borer, "Non-linear opto-electrical transfer functions for high dynamic range television," Tech. Rep. WP-283, BBC R\&D, London, UK, July 2014.

[4] Scott J. Daly and Xiaofan Feng, "Bit-depth extension using spatiotemporal microdither based on models of the equivalent input noise of the visual system," in Proc. SPIE, 2003, vol. 5008, pp. 455-466.

[5] L. G. Roberts, "Picture coding using pseudo-random noise," IRE Transactions on Information Theory, vol. IT, no. 8, pp. 145-154, Feb. 1962.

[6] T. K. Tan and Y. Suzuki, "Contouring artefact and solution," Tech. Rep. JCTVC-K0139, JCT-VC, Shanghai, CN, Oct. 2012, 11th meeting.

[7] K. Yoo, H. Song, and K. Sohn, "In-loop selective processing for contour artefact reduction in video coding," Electronics Letters, vol. 45, no. 20, pp. 1020-1022, Sept. 2009.

[8] S. Bhagavathy, J. Llach, and J. Zhai, "Multiscale probabilistic dithering for suppressing contour artifacts in digital images," IEEE Transactions on Image Processing, vol. 18, no. 9, pp. 1936-1945, Sept. 2009.

[9] X. Jin, S. Goto, and K. N. Ngan, "Composite model-based DC dithering for suppressing contour artifacts in decompressed video," IEEE Transactions on Image Processing, vol. 20, no. 8, pp. 2110-2121, Aug. 2011.

[10] Y. Wang, C. Abhayaratne, R. Weerakkody, and M. Mrak, "Multi-scale dithering for contouring artefacts removal in compressed UHD video sequences," in IEEE Global Conference on Signal and Information Processing (GlobalSIP), 2014, vol. 1, pp. 1-5.

[11] J. W. Lee, B. R. Lim, R.-H. Park, J.-S. Kim, and W. Ahn, “Two-stage false contour detection algorithm using re-quantization and directional contrast features and its application to adaptive false contour reduction," in International Conference on Consumer Electronics (ICCE), Jan. 2006, pp. 377-378.

[12] H.-R. Choi, J.W. Lee, R.-H. Park, and J.-S. Kim, "False contour reduction using directional dilation and edge-preserving filtering," IEEE Transactions on Consumer Electronics, vol. 52, no. 3, pp. 1099-1106, Aug. 2006.

[13] W. Ahn and J.-S. Kim, "Flat-region detection and false contour removal in the digital TV display," in IEEE International Conference on Multimedia and Expo (ICME), July 2005, pp. 1338-1341.

[14] S. J. Daly and X. Feng, "Decontouring: prevention and removal of false contour artifacts," in Proceedings of SPIE, 2004, vol. 5292, pp. $130-149$.

[15] F. Bossen, "Common HM test conditions and software reference configurations," Tech. Rep. JCTVC-L1100, JCT-VC, Geneva, CH, Jan. 2013, 12th meeting.

[16] K. McCann, C. Rosewarne, B. Bross, M. Naccari, K. Sharman, and G. J. Sullivan, "High efficiency video coding (HEVC) test model 16 (HM 16) improved encoder description," Tech. Rep. JCTVC-S1002, JCT-VC, Strasbourg, FR, Oct. 2014.

[17] G. Bjøntegaard, "Calculation of average PSNR differences between RD-curves," Tech. Rep. VCEG-M33, VCEG, Austin, TX, USA, Apr. 2001. 